\title{
LipF increases rifampicin and streptomycin sensitivity in a Mycobacterium tuberculosis surrogate
}

\author{
Ana Leticia Arriaga-Guerrero 1,2 , Carlos E. Hernández-Luna², Joyce Rigal-Leal², Rene J. Robles-González², \\ Laura Adiene González-Escalante ${ }^{1}$, Beatriz Silva-Ramírez ${ }^{3}$, Roberto Mercado-Hernández ${ }^{2}$, Javier Vargas-Villarreal ${ }^{4}$, \\ Mario Bermúdez de León ${ }^{1}$ and Katia Peñuelas-Urquides ${ }^{1 *}$ (D)
}

\begin{abstract}
Background: Mortality due to tuberculosis (TB) has increased due to the development of drug resistance, the mechanisms of which have not been fully elucidated. Our research group identified a low expression of lipF gene in Mycobacterium tuberculosis clinical isolates with drug resistance. The aim of this work was to evaluate the effect of lipase F (LipF) expression on mycobacterial drug resistance.

Results: The effects of expressing lipF from Mycobacterium tuberculosis in Mycobacterium smegmatis on resistance to antituberculosis drugs were determined with resazurin microtiter assay plate and growth kinetics. Functionality of ectopic LipF was confirmed. LipF expression reduced the rifampicin (RIF) and streptomycin (STR) minimum inhibitory concentration (MIC) from $3.12 \mu \mathrm{g} / \mathrm{mL}$ to $1.6 \mu \mathrm{g} / \mathrm{mL}$ and $0.25 \mu \mathrm{g} / \mathrm{mL}$ to $0.06 \mu \mathrm{g} / \mathrm{mL}$ respectively, moreover a reduced $M$. smegmatis growth in presence of RIF and STR compared with that of a control strain without LipF expression $(p<0.05$ and $p<0.01)$ was shown.

Conclusions: LipF expression was associated with increased RIF and STR sensitivity in mycobacteria. Reduced LipF expression may contribute to the development of RIF and STR resistance in Mycobacterium species. Our findings provide information pertinent to understanding mycobacterial drug resistance mechanisms.
\end{abstract}

Keywords: Mycobacterium, Tuberculosis, Rifampicin-resistance, Streptomycin-resistance, LipF, lipases

\section{Background}

Mycobacterium tuberculosis is the main causative agent of tuberculosis (TB), which is the leading cause of mortality due to infection worldwide. In 2017, there was an estimated of 10 million TB cases and 1.3 million deaths [1]. The first antibiotic discovered to treat tuberculosis in 1947 was streptomycin (STR) [2], this drug acts inhibiting protein synthesis through $30 \mathrm{~S}$ ribosomal subunit inhibition [3]. For many years this drug was used in

\footnotetext{
* Correspondence: katia.penuelasu@imss.gob.mx

1 Departamento de Biología Molecular, Centro de Investigación Biomédica del Noreste, Instituto Mexicano del Seguro Social, Monterrey, Nuevo León, México

Full list of author information is available at the end of the article
}

monotherapy in TB treatment therefore high drugresistance levels appeared and the incorporation of different antibiotics to the treatment scheme became necessary [4]. STR use is recommended as part of the second-line treatment regimen and only when amikacin is not available or its resistance had been confirmed [5]. Nowadays, the standard TB treatment includes antimicrobial drugs such as rifampicin (RIF), isoniazid (INH), pyrazinamide (PZA), and ethambutol (EMB) [1]. RIF is a semisynthetic molecule produced in Streptomyces mediterranei with broad spectrum antibacterial activity. Its mechanism of action consists in the inhibition of RNA polymerase activity by forming a stable complex

(c) The Author(s). 2020 Open Access This article is licensed under a Creative Commons Attribution 4.0 International License, which permits use, sharing, adaptation, distribution and reproduction in any medium or format, as long as you give appropriate credit to the original author(s) and the source, provide a link to the Creative Commons licence, and indicate if changes were made. The images or other third party material in this article are included in the article's Creative Commons licence, unless indicated otherwise in a credit line to the material. If material is not included in the article's Creative Commons licence and your intended use is not permitted by statutory regulation or exceeds the permitted use, you will need to obtain permission directly from the copyright holder. To view a copy of this licence, visit http://creativecommons.org/licenses/by/4.0/. The Creative Commons Public Domain Dedication waiver (http://creativecommons.org/publicdomain/zero/1.0/) applies to the data made available in this article, unless otherwise stated in a credit line to the data. 
with it $[6,7]$. Currently, RIF is considered to be the most effective first-line anti-TB drug and when administered with PZA the treatment regimen diminished to 6 months [8]. M. tuberculosis resistant to RIF and INH has become a serious problem. TB that is resistant to both drugs is defined as multidrug resistant (MDR)-TB [9]. Currently the TB epidemic is further exacerbated by the existence of MDR-TB. In 2017, there were approximately 558,000 new MDR-TB cases worldwide [1, 10]. Deficient treatment adherence by patients leads to selection pressure for drug-resistant (DR)-TB strains. The emergence and spread of drug resistance pathogens, particularly MDR-TB strains, pose a serious threat to human health worldwide [11]. Horizontal gene transfer has not been reported in M. tuberculosis, and MDR-TB has been generally associated with mutations. However mutations have not been identified in some MDR strains, which suggests that other mechanisms could be involved [12, 13]. A clinical MDR M. tuberculosis isolate was reported to have differential gene expression compared with that in the pansensitive $\mathrm{H} 37 \mathrm{Rv}$ strain. Notably, the MDR strain had lipF (Rv3487c) gene down-regulated [14]. This gene encodes for a lipase with phospholipase $\mathrm{C}$ and carboxylesterase activities and has particularly high activity with four-carbon para-nitrophenyl (pNP)derivate ester substrates $[15,16]$. Recently, lipases have been implicated in drug sensitivity and resistance [17, 18]. In a recent study of 24 clinical isolates of $M$. tuberculosis with varying drug resistance profiles and genetic backgrounds, lipF expression was found to be reduced in $\sim 90 \%$ of these resistance strains compared with that in the pansensitive reference strain H37Rv [19]. Although lipase $\mathrm{F}$ has been studied in $M$. tuberculosis virulence $[16,20,21]$; its role in drug resistance has not been addressed. Therefore, the aim of the present work was to evaluate the effect of lipF expression on drug resistance in a $M$. tuberculosis surrogate.

\section{Results}

Nucleotide sequence comparison between pansensitive and MDR strains

Nucleotide sequence analysis were performed on the promoter and coding sequences of lipF to determine whether differential expression between the pansensitive
H37Rv strain and the MDR CIBIN:UMF:15:99 clinical isolate, previously reported [14], could be due to mutations (Fig. 1). No sequence differences were found in the lipF promoter (477 bp), coding sequence (834 bp), or intergenic region (147 bp) between the two strains [Additional file 1], suggesting that the observed differential expression could involve other unknown regulation mechanisms.

\section{LipF expression in Mycobacterium smegmatis}

To elucidate the role of Lipase F in drug resistance, we evaluated the effects of overexpressing it in a $M$. tuberculosis surrogate, $M$. smegmatis. A pMV261-lipF containing a specific mycobacterial control region fused to the lipF coding sequence was constructed. Automated Sanger sequencing verified the fidelity of sequence (data not shown), which was confirmed to have no nucleotide alterations. Following separate transformation of pMV261 or pMV261-lipF into M. smegmatis ( $\mathrm{mc}^{2} 155$ strain), reverse-transcriptase (RT)polymerase chain reaction (PCR) analysis performed with lipF-specific primers confirmed that the lipF product (834 bp) was amplified in pMV261-lipF transformants. The pMV261-lipF transformants samples (Fig. 2 a) were treated with DNase I to eliminate bacterial genomic, and plasmidic DNA and RT were omitted in a control group to demonstrate that amplification was obtained solely from RNA (Fig. 2a, lanes 2 and 3). Western blot analysis with antiLipF polyclonal antibody (see Materials and methods) confirmed the expression of a $29-\mathrm{kDa}-\mathrm{lipF}$ protein product in M. smegmatis transformed with pMV261-lipF, but not in pMV261-transformed controls (Fig. 2 b).

Tween cleavage assays of enzyme activity were employed to probe functionality of LipF produced from pMV261-lipF transformants. We observed an 20\% increase in esterase activity (as indexed by Tween 20 hydrolysis) with protein extract from pMV261-lipF transformants compared with that from pMV261 control transformants not expressing LipF. Meanwhile we found a $14 \%$ increase in lipase activity (as indexing by Tween 80 hydrolysis) in LipF expressing transformants compared with that from control pMV261 M. smegmatis (Fig. 3). These results demonstrate that the recombinant LipF $M$. smegmatis has increased esterase and lipase activities.

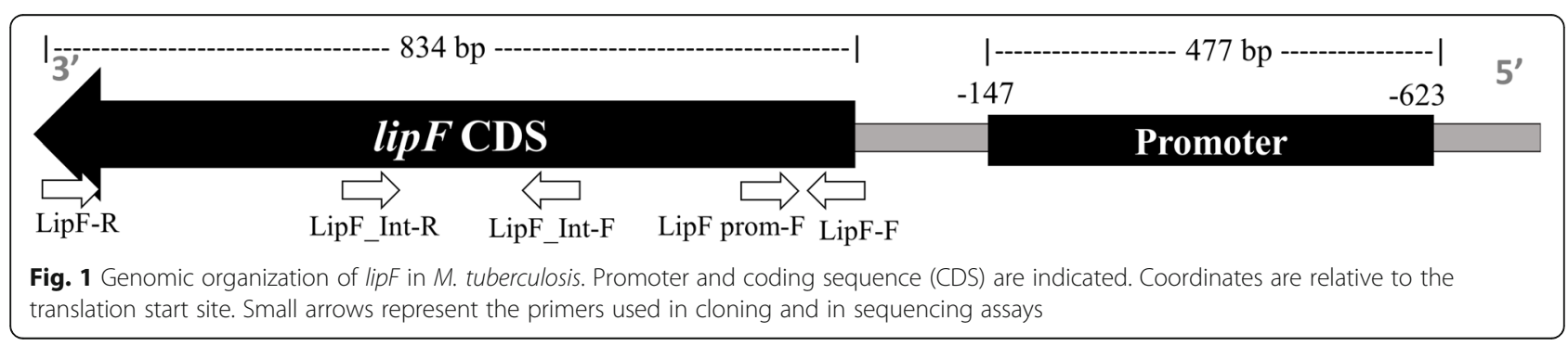




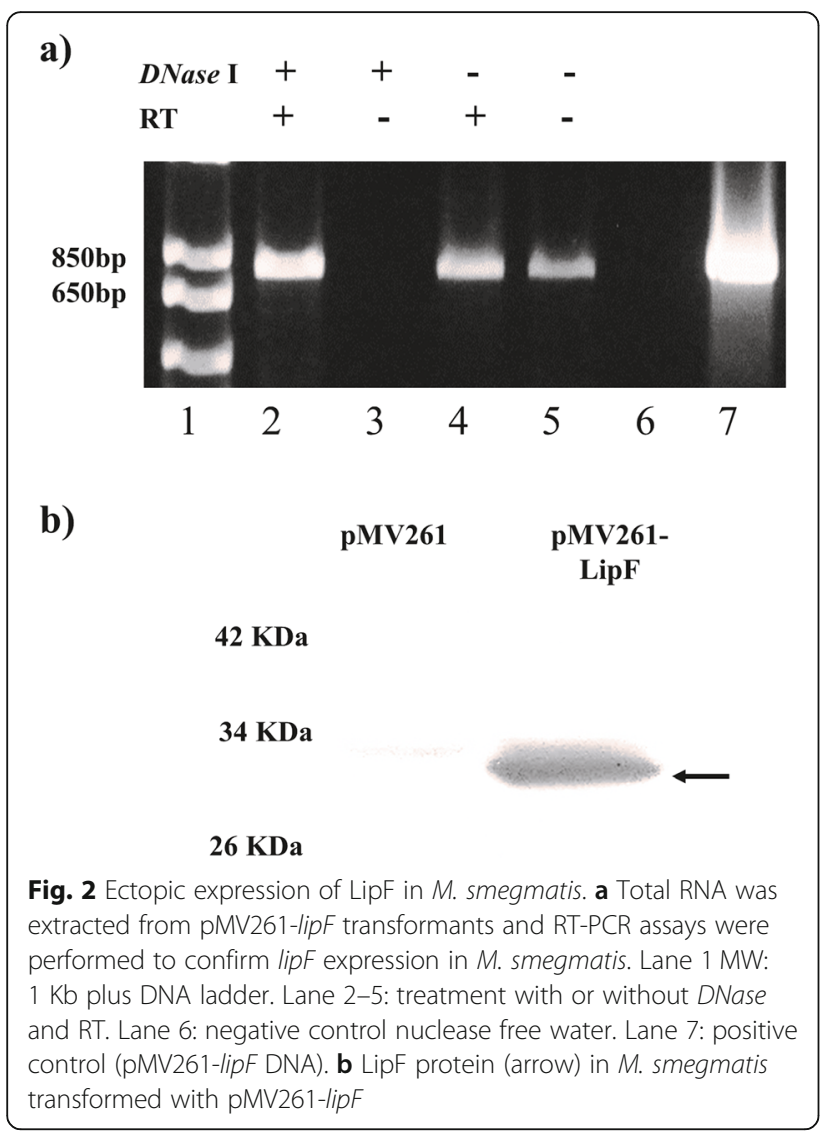

\section{Ectopic LipF expression modifies RIF and STR sensitivity} in $M$. smegmatis

To evaluate the involvement of LipF expression in firstline TB drug resistance phenomena, we determined minimum inhibitory concentrations (MICs) for multiple antibiotic agents in a resazurin microtiter assay plate (REMA). The pMV261 and pMV261-lipF transformants

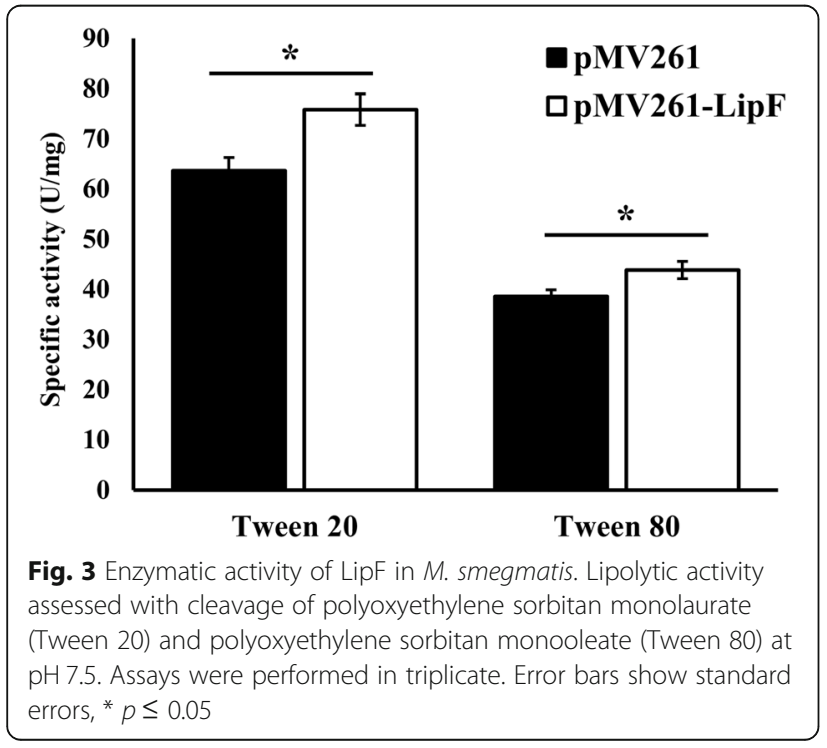

had no changes in MICs for INH and EMB $(32.0 \mu \mathrm{g} / \mathrm{mL}$ for $\mathrm{INH}$ and $0.5 \mu \mathrm{g} / \mathrm{mL}$ for EMB). Both transformants grew in presence of pyrazinamide (PZA) at all tested concentration (maximum $200 \mu \mathrm{g} / \mathrm{mL}$ ) (Table 1); PZA MIC for $M$. smegmatis has previously been shown to be $>2000 \mu \mathrm{g} / \mathrm{mL}$ [22]. However, pMV261-lipF transformant was more susceptible to RIF $(1.6 \mu \mathrm{g} / \mathrm{mL})$ than control transformant pMV261 $(3.12 \mu \mathrm{g} / \mathrm{mL})$, and STR $(0.06 \mu \mathrm{g} /$ $\mathrm{mL})$ versus control pMV261 $(0.25 \mu \mathrm{g} / \mathrm{mL})$ (Table 1$)$. Consistent with the aforementioned MIC results, bacterial growth kinetics assays with three RIF concentrations $(0.8 \mu \mathrm{g} / \mathrm{mL}, \quad 1.6 \mu \mathrm{g} / \mathrm{mL}$ and $3.2 \mu \mathrm{g} / \mathrm{mL})$ showed that growth was similar between the transformants pMV261 and pMV261-lipF M. smegmatis in presence of $3.2 \mu \mathrm{g} /$ $\mathrm{mL}$ and $0.8 \mu \mathrm{g} / \mathrm{mL}$ RIF in 7H9 medium (Fig. 4a). However, in presence of $1.6 \mu \mathrm{g} / \mathrm{mL}$ RIF we observed significantly reduced growth for pMV261-LipF compared to the observed for the pMV261 control $(p<0.05)$ (Fig. 4a). Regards to STR, bacterial growth kinetics with three different STR concentrations $(0.06 \mu \mathrm{g} / \mathrm{mL}, 0.12 \mu \mathrm{g} / \mathrm{mL}$ and $0.25 \mu \mathrm{g} / \mathrm{mL}$ ) showed a significant growth reduction in transformants pMV261- lipF M. smegmatis in presence of 0.06 and $0.12 \mu \mathrm{g} / \mathrm{mL}$ compared with the pMV261 control ( $p<0.05$ and $p<0.01$, respectively; Fig. $4 \mathrm{~b})$.

\section{Discussion}

In a previous study, LipF expression was diminished in a clinical MDR $M$. tuberculosis isolate relative to that of in a pansensitive H37Rv strain. To define if this differential expression is due to mutations, we examine the regulatory regions of $l i p F$, including the complete promoter regions that lies -147 to -623 nucleotides upstream of the translational start site of lipF, as well as lipF complete coding region from +1 to +834 and the intergenic region. The 477 base pair region of the promoter includes a 59 base pair minimal promoter locus responsible differential lipF expression under acid stress [20, 21]. We did not uncover any sequence differences between the promoters in a clinical MDR M. tuberculosis isolate and in the pansensitive $M$. tuberculosis reference strain. This result suggests that other mechanisms are involved in the differential expression of LipF in MDR M. tuberculosis.

Table 1 MICs of first-line TB drugs in M. smegmatis transformants

\begin{tabular}{lll}
\hline Drug & \multicolumn{2}{l}{ Minimum inhibitory concentration (MIC) } \\
\cline { 2 - 3 } & M. smegmatis pMV261 & M. smegmatis pMV261-LipF \\
\hline Rifampicin & $3.12 \mu \mathrm{g} / \mathrm{mL}$ & $1.6 \mu \mathrm{g} / \mathrm{mL}$ \\
Isoniazid & $32.0 \mu \mathrm{g} / \mathrm{mL}$ & $32.0 \mu \mathrm{g} / \mathrm{mL}$ \\
Ethambutol & $0.5 \mu \mathrm{g} / \mathrm{mL}$ & $0.5 \mu \mathrm{g} / \mathrm{mL}$ \\
Pyrazinamide & $>200 \mu \mathrm{g} / \mathrm{mL}$ & $>200 \mu \mathrm{g} / \mathrm{mL}$ \\
Streptomycin & $0.25 \mu \mathrm{g} / \mathrm{mL}$ & $0.06 \mu \mathrm{g} / \mathrm{mL}$ \\
\hline
\end{tabular}




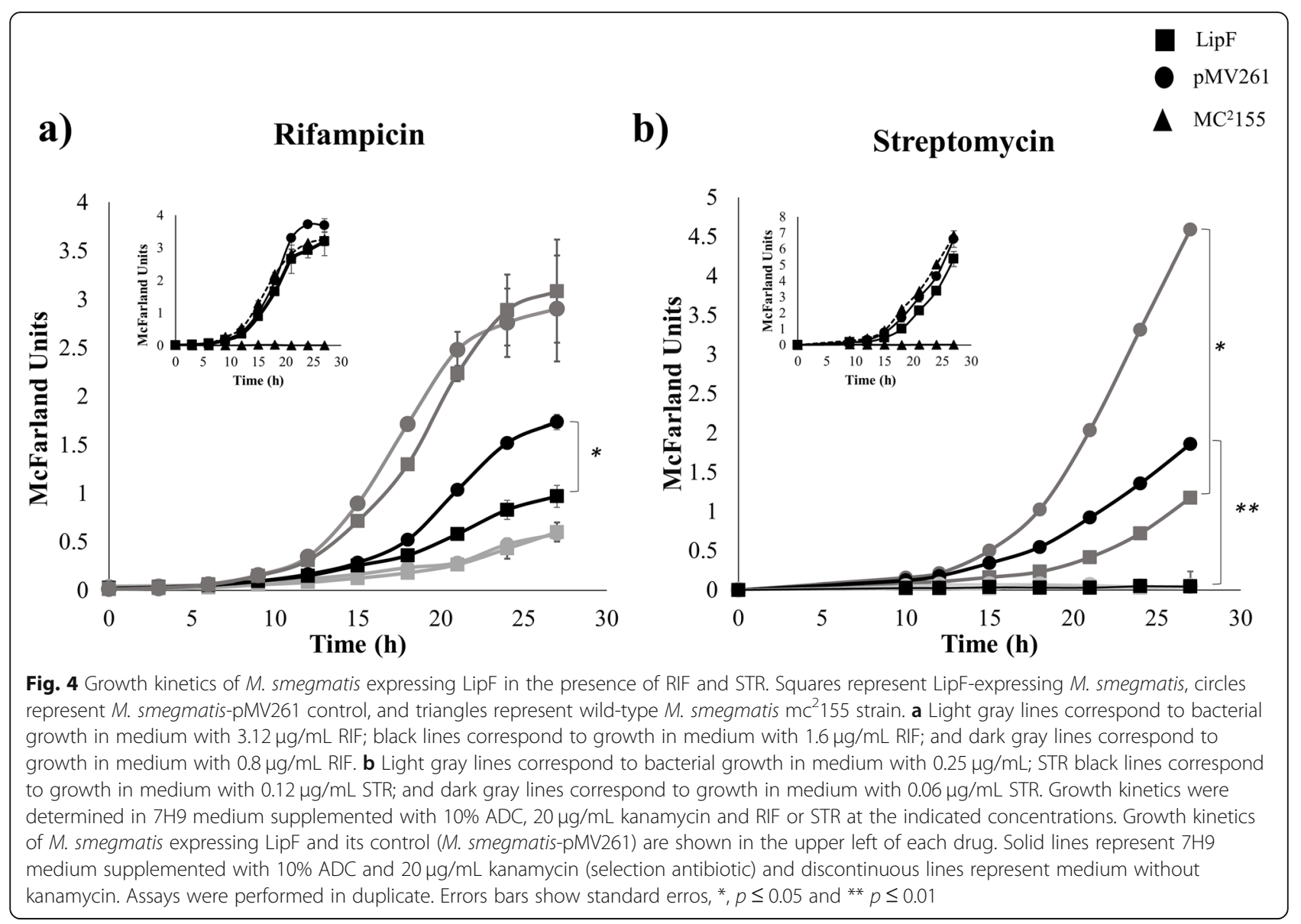

We selected $M$. smegmatis as a surrogate species for M. tuberculosis because it can be handled safely, and it grows fast. Heterologous expression of LipF in M. smegmatis was confirmed with the affirmation of the presence of lipF transcript and an associated protein of the expected molecular weight for $\operatorname{LipF}(29 \mathrm{kDa})$ [15]. The functionality of this protein was analyzed in Tweencleavage enzyme assays. Tween cleavage assays which have been used extensively to determine lipase/esterase activity, have been reported to be 36 times more sensitive than the titrimetric assay (using triacetic acids) and four times more sensitive than spectrophotometry with $p$-NP palmitate [17, 23-25]. Our finding of increased esterase activity in the LipF-expressing strain is consistent with prior work demonstrating esterase activity for LipF, which prefers water-soluble short chain fatty acid esters as substrates and has a high affinity for glycerol acetate and $p$-NP acetate. LipF has not been shown previously to act on triacylglycerides or $p$-NP esters with longchain fatty acids, such as tricaprin and $p$-NP caprate [15]. Notably, we found a $14 \%$ increase in lipase activity acting on long-chain fatty acids. Therefore, the present data confirmed lipase and esterase activities of the recombinant LipF.
Determination of MICs for bacteria is a gold standard method in antimicrobial susceptibility wherein a MIC is defined as the lowest concentration inhibits detectable growth of a microorganism over predetermined time period [26]. The presently determined MICs for wildtype $M$. smegmatis were different from previously reported MICs [27]. This discordance could be due to the use of parafilm to seal plates for $40 \mathrm{~h}$ incubation in previous assays; sealing may reduce oxygen availability, which could slow growth of mycobacteria and increase their susceptibility to drugs. Regardless, the MICs obtained in the present study were reproducible. Compared to controls, $M$. smegmatis transformants expressing LipF had a reduced RIF and STR MICs but unaltered EMB and INH MICs. Consistent with our RIF and STR MICs results, a bacterial growth assay showed slower growth in LipF-expressing transformants cultured in medium containing $1.6 \mu \mathrm{g} / \mathrm{mL}$ RIF $(p<0.05)$, and in cultures in medium containing $0.12 \mu \mathrm{g} / \mathrm{mL}$ and $0.06 \mu \mathrm{g} / \mathrm{mL}$ STR $(p<0.01$ and $p<0.05$, respectively). These results indicate that LipF expression in M. smegmatis confers an enhance sensitivity to RIF and STR.

Previously, Rv0183, LipX, and LipG lipases, these last from the Lip family, have been related to drug resistance. 
Disruption of a Rv0183 homolog in M. smegmatis modified sensitivity to RIF and INH [28]. LipX overexpression in $M$. smegmatis was shown to increase resistance to the first-line anti-TB drugs INH, EMB, and RIF, whereas LipG disruption in $M$. smegmatis was shown to augment RIF and INH resistance [17, 18]. It is unclear whether enhance RIF sensitivity in M. smegmatis expressing LipF in our study can be attribute to a direct interaction between RIF and LipF. A molecular docking experiment show only one possible mode of interaction, which was weak (hydrogen bond with a root-mean-square deviation value <3) (data not shown). Meanwhile LipX and LipG which can modify mycobacterial drug resistance alter the composition of cell walls by increasing their glycopeptidelipid contents $[17,18]$. Being a non-polar molecule, RIF can enter the cell through passive diffusion [29]. Interestingly, a RIF persistent $M$. tuberculosis strain was recently reported to have a thick outer layer, atypically abundant polysaccharides, whose presence increases hydrophobicity and reduces efflux pumps-independent RIF influx [30]. Thus, changes in cell wall composition and polarity can alter the access of antibiotic agents, such as RIF, to the inside of bacteria. Resistance to aminoglycosides has been associated to bacterial cell membranes alterations regarding permeability [31]; a diffusion of STR when porins were inhibited in E. coli have been demonstrated [32]. Moreover some $M$. tuberculosis clinical isolates resistant to STR has been associated with a lower cell wall permeability [33]. Given this potential, we suggest that the esterase and lipase activities of LipF could, potentially increase mycobacterial susceptibility to RIF and STR by modifying mycobacterial cell walls in a manner that enable entry of these drugs into cell.

Overexpression of rроB has been implicated in RIF resistance in Mycobacterium [34]. In order to demonstrate if rpoB expression is involved in rifampicin and streptomycin susceptibility we performed an expression analysis using a RT-PCR assay and a semi-quantitative densitometric analysis and we did not find differences in expression of rроB gene between strains expressing LipF and its empty vector control (data no shown).

Although inhibition of lipF has been shown to modify M. tuberculosis growth in a previous study [35]; we did not observe effects of LipF expression on M. smegmatis growth in this study (Fig. S1). Rather, we found that, ectopic expression of LipF increased $M$. smegmatis sensitivity to RIF and STR suggesting that reduced-lipF expression may contribute to the development of RIF and STR resistance in mycobacteria. More studies are needed to demonstrate LipF participation in drug resistance, to clarify the mechanism by which LipF may contribute to RIF and STR resistance. Furthermore, more analyses with second line drugs are needed to identify other potential roles of lipases in drug resistance.

\section{Conclusions}

In conclusion, we demonstrated that ectopic expression of LipF increases sensitivity to RIF and STR. These results suggest that low LipF expression in M. tuberculosis may be an important enabler for RIF and STR resistance.

\section{Methods}

\section{Strains and growth conditions}

DH5 $\alpha$ strain Escherichia coli (E. coli) was used for construct transformations, and $\mathrm{mc}^{2} 155$ M. smegmatis strain was used as a protein expression surrogate for $M$. tuberculosis. H37Rv strain M. tuberculosis (GenBank: AL123456.3) and the clinical CIBIN:UMF:15:99 MDR M. tuberculosis isolate were obtained from a repository located in the Centro de Investigación Biomédica del Noreste, Instituto Mexicano del Seguro Social.

E. coli was grown in LB medium supplemented with $75 \mu \mathrm{g} / \mathrm{mL}$ kanamycin for transformant selection, liquid or solid agar. M. smegmatis was grown in Middlebrook 7H9 medium supplemented with $10 \%$ album-dextrose-catalase (ADC) and $0.05 \%$ Tween $80 ; 20 \mu \mathrm{g} / \mathrm{mL}$ kanamycin was added for transformant selection. E. coli and M. smegmatis were incubated at $37^{\circ} \mathrm{C}$ for $24 \mathrm{~h}$ for liquid cultures and $3 \mathrm{~d}$ for solid cultures. M. tuberculosis was grown as previously reported [14]. Briefly, Middlebrook 7H9 medium supplemented with $10 \%$ oleic acid, albumin, dextrose, and catalase (OADC). These cultures were incubated at $37^{\circ} \mathrm{C}$ in a $5 \%$ $\mathrm{CO}_{2}$ atmosphere until they reached a turbidity equivalent to the $1.0 \mathrm{McFarland}$ standard. A $100 \mu \mathrm{L}$ aliquot was inoculated in $10 \mathrm{~mL}$ of the aforementioned supplemented Middlebrook 7H9 medium. Liquid cultures were incubated at $37^{\circ} \mathrm{C}$ in a $5 \% \mathrm{CO}_{2}$ atmosphere with constant orbital shaking $(300 \mathrm{rpm})$ until they reached $\log$ phase growth (i.e., 3.0-4.0 McFarland).

For growth curves, pMV261 and pMV261-lipF transformants of $M$. smegmatis were grown until they reached 1.0 McFarland unit. Then, they were reinoculated in fresh medium $\left(3.08 \times 10^{5}\right.$ bacteria $\left./ \mathrm{mL}\right)$ with RIF $(0.8 \mu \mathrm{g} / \mathrm{mL}, 1.6 \mu \mathrm{g} / \mathrm{mL}$ and $3.12 \mu \mathrm{g} / \mathrm{mL})$ or STR $(0.25 \mu \mathrm{g} / \mathrm{mL}, 0.12 \mu \mathrm{g} / \mathrm{mL}$ and $0.06 \mu \mathrm{g} / \mathrm{mL})$ and $20 \mu \mathrm{g} / \mathrm{mL}$ kanamycin. Cultures were incubated at $37{ }^{\circ} \mathrm{C}$ with constant shaking for $27 \mathrm{~h}$, and McFarland units were measured every $3 \mathrm{~h}$ with a DensiCHECK ${ }^{\mathrm{m}}$ Plus nephelometer (BioMérieux, Marcy-l'Étoile, France). All experiments were performed in duplicate, and average values were used to generate the growth kinetic curves.

\section{MIC determination}

MICs for the first line TB drugs (rifampicin, isoniazid, ethambutol and pyrazinamide) and STR were determined with REMA method, previously described [27]. Briefly, 96-well plates were filled with $50 \mu \mathrm{L}$ of $7 \mathrm{H} 9$ media (for PZA the medium was acidified at $\mathrm{pH}$ 5.5) except for the first column. Antibiotic concentration stocks 
were prepared at double the needed concentrations and $100-\mu \mathrm{L}$ were added to each well of the first column, and then diluted serially by half concentration by mixing with media in the subsequent wells. The last columns were used as no-antibiotic controls. Plates were inoculated with $2.77 \times 10^{4}$ bacteria $/ \mathrm{mL}$ and incubated at $37^{\circ} \mathrm{C}$ under constant agitation for $40 \mathrm{~h}$. Subsecuently, $30 \mu \mathrm{L}$ of resazurin reagent $(20 \mu \mathrm{g} / \mathrm{mL})$ was added to each well and incubated for $6 \mathrm{~h}$. MICs were then determined when a change of color from blue to pink was observed.

\section{Plasmid construction}

Genomic DNA from M. tuberculosis (H37Rv strain) was used as an amplification template. To amplify an 834-base pair product, the following specific primers for the lipF coding sequence were used: forward 5 '-CAC CGG ATC CAA TGC GTG CGC CTG GGG TG-3'; and reverse 5' GGA TCC CTA GAT AGG CGA CCT GTC CAA AC-3' (underlined sequence corresponds to $\mathrm{BamHI}$ recognition site). PCR was performed in a final volume of $25 \mu \mathrm{L}$ with the following constituents: Taq DNA polymerase buffer $1 \times, 2 \mathrm{mM}$ dNTPs, $3.5 \mathrm{mM} \mathrm{MgCl} 2,5 \% \mathrm{DMSO}$, and $1 \mathrm{U}$ Taq DNA. The PCR program was: one 5-min denaturation period $95^{\circ} \mathrm{C}$, thirty 30 s cycles of denaturation at 95 ${ }^{\circ} \mathrm{C}$, primer annealing at $58{ }^{\circ} \mathrm{C}$ for $30 \mathrm{~s}$, polymerization at $72{ }^{\circ} \mathrm{C}$ for $30 \mathrm{~s}$, and a final $5 \mathrm{~min}$ step at $72{ }^{\circ} \mathrm{C}$. The PCR products were visualized by electrophoresis in a $1 \%(\mathrm{w} / \mathrm{v})$ agarose gel stained with Gel red (Biotium, Hayward, USA). The lipF PCR product was inserted in pET101/D plasmid (Invitrogen, USA), as a transition vector.

For lipF expression in M. smegmatis, the mycobacterial pMV261 vector was used. The pET101/D-LipF construct and pMV261 vector were digested with BamHI enzyme, and ligation of lipF in pMV261 was performed with T4 DNA ligase (Invitrogen, Carlsbad, CA). Positive clones, defined as having the gene inserted in the sense direction in PMV261-lipF, were confirmed by PCR amplification with a primer that aligns in a vector region (pMV261 Forward 5'-GTTGTAGTGCTTGTGTGGCA$\left.3^{\prime}\right)$ and the lipF reverse primer and DNA sequencing.

\section{RNA and protein expression analysis}

M. smegmatis $\mathrm{mc}^{2} 155$ cells were transformed by electroporation with pMV261 or pMV261-lipF construct and incubated at $37^{\circ} \mathrm{C}$ on $7 \mathrm{H} 10$ agar plates containing $20 \mu \mathrm{g} / \mathrm{mL}$ kanamycin. After $3 \mathrm{~d}$ of incubation, single colonies were isolated and grown in $10 \mathrm{~mL}$ of $7 \mathrm{H} 9$ supplemented with $10 \%$ ADC, $0.05 \%$ Tween 80 and $20 \mu \mathrm{g} / \mathrm{mL}$ kanamycin. The culture conditions were $37^{\circ} \mathrm{C}$ with of constant shaking at $300 \mathrm{rpm}$ for $20 \mathrm{~h}$. Then, total RNA was extracted with TRIzol reagent (Invitrogen, Carlsbad, CA) according to manufacturer's instructions. Briefly, $10 \mathrm{~mL}$ of mycobacterial culture was centrifuged for 20 min at $25,000 \times \mathrm{g}$ at $4{ }^{\circ} \mathrm{C}$; after supernatant removal, the bacterial pellet was suspended in $1 \mathrm{~mL}$ of TRIzol reagent and incubated for $20 \mathrm{~min}$ at room temperature. Samples were then transferred to Fast Prep tubes containing Lysin Matrix B (MP Biomedicals, Solon, OH. USA) and processed for three $20 \mathrm{~s}$ cycles at a velocity setting of 6 , with 3 min cooling on ice period between cycles. RNA was precipitated with chloroform and isopropyl alcohol and then, washed with ethanol. Finally, RNA was resuspended in Tris-EDTA buffer (Promega, Madison, WI. USA) and treated with DNase I (Invitrogen, Carlsbad, CA. USA).

The purity and concentration of RNA were estimated by a Nanodrop spectrophotometer. Synthesis of cDNA was carried out with M-MLV RT in the presence of random primers (Invitrogen, Carlsbad, CA. USA) following the conditions specified by the manufacturer. RT reaction mix $1(0.6-2 \mu \mathrm{g}$ of total RNA, random primers and deoxynucleoside triphosphate) was incubated at $65^{\circ} \mathrm{C}$ for $5 \mathrm{~min}$ and then cooled on ice. Reaction mix 2 (first strand buffer $1 \times$, DTT and RNase OUT) was added to mix 1 and incubated at $37^{\circ} \mathrm{C}$ for $2 \mathrm{~min}$. Then M-MLV RT was added. The final reaction was incubated at $25^{\circ} \mathrm{C}$ for $10 \mathrm{~min}$, followed by $37^{\circ} \mathrm{C}$ for $50 \mathrm{~min}$, and then $70^{\circ} \mathrm{C}$ for $15 \mathrm{~min}$.

For western blotting, specific antibodies against LipF were designed (Genemed Synthesis, Inc., San Antonio, TX). For protein extraction, frozen bacteria stocks were inoculated in $3 \mathrm{~mL}$ of $7 \mathrm{H} 9$ media supplemented with $10 \% \mathrm{ADC}, 0.05 \%$ Tween 80 and $20 \mu \mathrm{g} / \mathrm{mL}$ kanamycin. After $20 \mathrm{~h}$, an inoculum of $100 \mu \mathrm{L}$ was transferred into $30 \mathrm{~mL}$ of $7 \mathrm{H} 9$ medium. The cultures were incubated at $37^{\circ} \mathrm{C}$ for $20 \mathrm{~h}$, centrifuged, and the bacteria pellets were washed in $1 \times$ phosphate buffered saline to eliminate albumin residue. Bacterial lysis was performed with a FastPrep instrument (MP Biomedicals, Solon, OH.USA), wherein bacteria pellets were dissolved in $500 \mu \mathrm{L}$ of lysis buffer $(150 \mathrm{mM} \quad \mathrm{NaCl}, 1 \mathrm{mM} \quad \beta$ mercaptoethanol, $\mathrm{pH} 8.15$ and proteases inhibitors) and transferred to Fast Prep tubes containing Matrix B (MP Biomedicals, Solon, OH. USA). Cell lysis was obtained using three 20 s cycles at a velocity setting of 6 , and samples were cooled on ice between each cycle. The supernatant was collected after centrifugation at $15,294 \times \mathrm{g}$ (5430R Eppendorf) for $3 \mathrm{~min}$ and stored to $-20^{\circ} \mathrm{C}$ until use.

\section{Enzymatic activity}

M. smegmatis pMV261 and M. smegmatis pMV261-lipF were grown in $20 \mathrm{~mL}$ of $7 \mathrm{H} 9$ medium supplemented with $10 \%$ ADC, $0.05 \%$ Tween 80 and $20 \mu \mathrm{g} / \mathrm{mL}$ kanamycin until the cultures reached 4.0 McFarland units. Cultures were centrifuged, washed and resuspended in $800 \mu \mathrm{L}$ of lysis buffer as described previously [17] (50 $\mathrm{mM}$ Tris- $\mathrm{Cl}$ of $\mathrm{pH} 8.0,300 \mathrm{mM} \mathrm{NaCl}$ and protease inhibitors). Bacteria lysis was performed as mentioned 
above and protein concentration was determined by the Bradford method (Biorad, CA, USA).

Lipolytic activity was determined as described previously [17]. Briefly, a reaction mixture was prepared in $50 \mathrm{mM}$ Tris, $33 \mathrm{mM} \mathrm{CaCl}$, $0.33 \%$ Tween 80 or Tween 20 [pH 7.5] and $40 \mu \mathrm{g}$ cell lysate. This reaction mixture was incubated at $35^{\circ} \mathrm{C}$ for $30 \mathrm{~min}$. When the incubation was finished, $200 \mu \mathrm{L}$ were transferred to a 96-well microwell plate (Corning, NY, USA), and turbidity was assessed by measuring absorbance at $405 \mathrm{~nm}$. Enzymatic activity was reported as $\mathrm{U} / \mathrm{mg}$ of lysate, where $1 \mathrm{U}$ is defined as the amount of enzyme required to induce a change in absorbance of 0.01 under the assay conditions. Assays were performed in triplicate.

\section{Sequencing and analysis}

Mycobacterial DNA was isolated by the cetyltrimethylammonium bromide (CTAB) method as described in Peñuelas-Urquides et al. (2013) [14]. Automated Sanger sequencing was performed with $M$. tuberculosis H37Rv and CIBIN:UMF:15:99 genomic DNA. The purified PCR products were sequenced with a BigDye ${ }^{\mathrm{T} x}$ Terminator v3.1 Cycle Sequencing Kit following the manufacturer's instruction in a 3130xl Genetic Analyzer (Applied Biosystems, Foster City, CA, USA) with the following primers: LipF promoter, forward 5'-CCG TGC AAA TAG AGC ACC AC-3' (LipF Prom-F); LipF internal forward (LipF_Int-F), 5'-AAA CGT GAA TAA GTG TCG GC-3', and LipF internal reverse (LipF_Int-R) 5' - CAG TGC GAC GAC GAG AAA-3'(Fig. 1). The sequence analysis was performed Seqscape v2.7 software with the GenBank reference NC_000962.3.

\section{Statistics}

Growth curves were developed based on biological duplicates, and statistical analyses were performed through slope of the line comparisons. Enzymatic activity was determined in technical triplicates assays and analyzed with Mann-Whitney tests in SPSS v.20 (IBM). $P$ values $\leq 0.05$ were considered significant.

\section{Supplementary information}

Supplementary information accompanies this paper at https://doi.org/10. 1186/s12866-020-01802-X.

Additional file 1. LipF sequence comparison between M. tuberculosis H37Rv and clinical CIBIN:UMF:15:99. H37Rv isolate. GenBank: CP009480.1 was used for reference. The LipF coding sequence is underlined, and the LipF promoter sequence is marked in gray. Sequences were obtained through automated sequencing and aligned in CLC sequence viewer 8.0 software.

Additional file 2: Fig. S1. Growth kinetics of M. smegmatis expressing LipF in a) RIF and b) STR assays. Growth of strains represent the control in medium without RIF and STR. Solid lines represent $7 \mathrm{H} 9$ medium supplemented with $10 \%$ ADC and $20 \mu \mathrm{g} / \mathrm{mL}$ kanamycin (selection antibiotic) and discontinuous lines represent medium without kanamycin.
Squares represent LipF-expressing M. smegmatis, circles represent $M$. smegmatis-pMV261 control, and triangles represent wild-type M. smegmatis $m c^{2} 155$ strain.

\section{Abbreviations}

TB: Tuberculosis; STR: Streptomycin; EMB: Ethambutol; RIF: Rifampicin; INH: Isoniazid; PZA: Pyrazinamide; MDR: Multidrug resistance; ADC: Albumin, dextrose and catalase; OADC: Oleic acid, albumin, dextrose and catalase; MIC: Minimal inhibitory concentration; REMA: Resazurin microtiter assay plate; pNP: $p$-Nitrophenyl

\section{Acknowledgements}

This work was supported by Instituto Mexicano del Seguro Social (FIS/IMSS/ PROT/G17-2/1734). We are grateful to C.P. Gabriela Mendez for the clerical work. We thank Dr. William Jacobs Jr. for providing pMV261 vector, Dr. Brian Weinrick for the constructive criticism of our manuscript, Ana Laura Granados-Tristán for counseling in bioinformatic assays, and Aldo Herrera for his help in molecular docking analysis. ALAG was recipient of CONACYT (574550) and IMSS (97208466) scholarships.

\section{Authors' contributions}

ALAG performed the assays, the analysis of data and co-drafted the manuscript, JRL performed the assays and the analysis of data and revised the manuscript, CEHL, LAGE, JW and RJRG performed analysis of data and revised the manuscript, BSR revised the manuscript, $\mathrm{RMH}$ performed statistical analysis, MBL participated in the design of the study, analysis of data and codrafted the manuscript, KPU performed the conception and design of study, the analysis of data and co-drafted the manuscript. The author(s) read and approved the final manuscript.

\section{Funding}

This work was supported by Instituto Mexicano del Seguro Social (FIS/IMSS/ PROT/G17-2/1734). The entity that financed this work did not participate in the design of the study, collection, analysis, and interpretation of the data, nor in writing of the version submitted for publication.

\section{Availability of data and materials}

Please contact corresponding author for data request.

Ethics approval and consent to participate

This study was approved by the National Commission for Scientific Research belonging to Instituto Mexicano del Seguro Social (Register 2016-785-105).

Consent for publication

Not Applicable.

\section{Competing interests}

The authors declare that they have no competing interests.

\section{Author details}

${ }^{1}$ Departamento de Biología Molecular, Centro de Investigación Biomédica del Noreste, Instituto Mexicano del Seguro Social, Monterrey, Nuevo León, México. ${ }^{2}$ Facultad de Ciencias Biológicas, Universidad Autónoma de Nuevo León, San Nicolás de los Garza, Nuevo León, México. ${ }^{3}$ Departamento de Inmunogenética, Centro de Investigación Biomédica del Noreste, Instituto Mexicano del Seguro Social, Monterrey, Nuevo León, México. ${ }^{4}$ Departamento de Biología Celular, Centro de Investigación Biomédica del Noreste, Instituto Mexicano del Seguro Social, Monterrey, Nuevo León, México.

Received: 29 October 2019 Accepted: 23 April 2020

Published online: 25 May 2020

References

1. WHO: Global Tuberculosis Report 2018. 2018.

2. Bloom BR, Murray CJ. Tuberculosis: commentary on a reemergent killer. Science. 1992;257(5073):1055-64.

3. Janin YL. Antituberculosis drugs: ten years of research. Bioorg Med Chem. 2007;15(7):2479-513.

4. Honore N, Cole ST. Streptomycin resistance in mycobacteria. Antimicrob Agents Chemother. 1994;38(2):238-42. 
5. WHO consolidated guidelines on drug-resistant tuberculosis treatment. WHO consolidated guidelines on drug-resistant tuberculosis treatment. Geneva; 2019

6. Mukherjee, A, Lodha, R, Kabra, SK. Pharmacokinetics of first-line antitubercular drugs. Indian J Pediatr. 2019;86(5),468-78.

7. Brunton LL, Hilal-Dandan R, Knollmann BC. Goodman \& Gilman's: The pharmacological basis of therapeutics, 13e. In: Shanahan JF, Lebowitz H, editors. USA: McGraw-Hill Education; 2018. Chapter 60.

8. A controlled trial of 6 months' chemotherapy in pulmonary tuberculosis. Final report: results during the 36 months after the end of chemotherapy and beyond. British Thoracic Society. Br J Dis Chest. 1984;78(4):330-6.

9. Toosky M, Javid B. Novel diagnostics and therapeutics for drug-resistant tuberculosis. Br Med Bull. 2014;110(1):129-40.

10. Shehzad A, Rehman G, UI-Islam M, Khattak WA, Lee YS. Challenges in the development of drugs for the treatment of tuberculosis. Braz J Infect Dis. 2013;17(1):74-81

11. LoBue P. Extensively drug-resistant tuberculosis. Curr Opin Infect Dis. 2009, 22(2):167-73.

12. Chaoui I, Sabouni R, Kourout M, Jordaan AM, Lahlou O, Elouad R, Akrim M, Victor TC, El Mzibri M. Analysis of isoniazid, streptomycin and ethambutol resistance in Mycobacterium tuberculosis isolates from Morocco. J Infect Dev Ctries. 2009;3(4):278-84.

13. Nguyen L. Antibiotic resistance mechanisms in M. tuberculosis: an update. Arch Toxicol. 2016;90(7):1585-604.

14. Penuelas-Urquides K, Gonzalez-Escalante L, Villarreal-Trevino L, Silva-Ramirez B, Gutierrez-Fuentes DJ, Mojica-Espinosa R, Rangel-Escareno C, UribeFigueroa L, Molina-Salinas GM, Davila-Velderrain J, et al. Comparison of gene expression profiles between pansensitive and multidrug-resistant strains of Mycobacterium tuberculosis. Curr Microbiol. 2013;67(3):362-71.

15. Zhang M, Wang JD, Li ZF, Xie J, Yang YP, Zhong Y, Wang HH. Expression and characterization of the carboxyl esterase Rv3487c from Mycobacterium tuberculosis. Protein Expr Purif. 2005;42(1):59-66.

16. Srinivas M, Rajakumari S, Narayana Y, Joshi B, Katoch VM, Rajasekharan R, Balaji KN. Functional characterization of the phospholipase $C$ activity of Rv3487c and its localization on the cell wall of Mycobacterium tuberculosis. J Biosci. 2008:33(2):221-30

17. Singh P, Rao RN, Reddy JR, Prasad RB, Kotturu SK, Ghosh S, Mukhopadhyay S. PE11, a PE/PPE family protein of Mycobacterium tuberculosis is involved in cell wall remodeling and virulence. Sci Rep. 2016;6:21624.

18. Santucci P, Point V, Poncin I, Guy A, Crauste C, Serveau-Avesque C, Galano JM, Spilling CD, Cavalier JF. Canaan S: LipG a bifunctional phospholipase/ thioesterase involved in mycobacterial envelope remodeling. Biosci Rep. 2018;38(6).

19. Gonzalez-Escalante L, Penuelas-Urquides K, Said-Fernandez S, Silva-Ramirez B, Bermudez de Leon M. Differential expression of putative drug resistance genes in Mycobacterium tuberculosis clinical isolates. FEMS Microbiol Lett 2015;362(23):fnv194.

20. Richter L, Tai W, Felton J, Saviola B. Determination of the minimal acidinducible promoter region of the lipF gene from Mycobacterium tuberculosis. Gene. 2007;395(1-2):22-8.

21. Gonzales M, Saviola B. Mutational analysis of the -10 region from the Mycobacterium tuberculosis lipF promoter. Mol Biol Rep. 2009;36(6):1225-9.

22. Boshoff HI, Mizrahi V. Purification, gene cloning, targeted knockout, overexpression, and biochemical characterization of the major pyrazinamidase from Mycobacterium smegmatis. J Bacteriol. 1998;180(22): 5809-14.

23. Gupta R, Rathi P, Gupta N, Bradoo S. Lipase assays for conventional and molecular screening: an overview. Biotechnol Appl Biochem. 2003;37(Pt 1): 63-71.

24. Pratt J, Cooley JD, Purdy CW, Straus DC. Lipase activity from strains of Pasteurella multocida. Curr Microbiol. 2000:40(5):306-9.

25. Plou FJ, Ferrer M, Nuero OM, Calvo MV, Alcalde M, Reyes F, Ballesteros A Analysis of tween 80 as an esterase/lipase substrate for lipolytic activity assay. Biotechnol Tech. 1998;12(3):183-6.

26. Andrews JM. Determination of minimum inhibitory concentrations. J Antimicrob Chemother. 2001:48(Suppl 1):5-16.

27. Agrawal P, Miryala S, Varshney U. Use of Mycobacterium smegmatis deficient in ADP-ribosyltransferase as surrogate for Mycobacterium tuberculosis in drug testing and mutation analysis. PLoS One. 2015;10(4): e0122076
28. Dhouib R, Laval F, Carriere F, Daffe M, Canaan S. A monoacylglycerol lipase from Mycobacterium smegmatis involved in bacterial cell interaction. J Bacteriol. 2010;192(18):4776-85.

29. Piddock $\amalg$, Williams KJ, Ricci V. Accumulation of rifampicin by Mycobacterium aurum, Mycobacterium smegmatis and Mycobacterium tuberculosis. J Antimicrob Chemother. 2000;45(2):159-65.

30. Sebastian Jees SS, Parthasarathi A. Reduced permeability to rifampicin by capsular thickening as a mechanism of antibiotic persistence in Mycobacterium tuberculosis: bioRxlv; 2019.

31. Delcour AH. Outer membrane permeability and antibiotic resistance. Biochim Biophys Acta. 2009:1794(5):808-16.

32. Nakae R, Nakae T. Diffusion of aminoglycoside antibiotics across the outer membrane of Escherichia coli. Antimicrob Agents Chemother. 1982;22(4): $554-9$.

33. Lambert PA. Cellular impermeability and uptake of biocides and antibiotics in gram-positive bacteria and mycobacteria. J Appl Microbiol. 2002; 92(Suppl):46S-54S

34. Zhu JH, Wang BW, Pan M, Zeng YN, Rego H, Javid B. Rifampicin can induce antibiotic tolerance in mycobacteria via paradoxical changes in rpoB transcription. Nat Commun. 2018;9(1):4218.

35. DeJesus MA, Gerrick ER, Xu W, Park SW, Long JE, Boutte CC, Rubin EJ, Schnappinger D, Ehrt S, Fortune SM, et al. Comprehensive Essentiality Analysis of the Mycobacterium tuberculosis Genome via Saturating Transposon Mutagenesis. MBio. 2017:8(1).

\section{Publisher's Note}

Springer Nature remains neutral with regard to jurisdictional claims in published maps and institutional affiliations.
Ready to submit your research? Choose BMC and benefit from:

- fast, convenient online submission

- thorough peer review by experienced researchers in your field

- rapid publication on acceptance

- support for research data, including large and complex data types

- gold Open Access which fosters wider collaboration and increased citations

- maximum visibility for your research: over $100 \mathrm{M}$ website views per year

At $\mathrm{BMC}$, research is always in progress.

Learn more biomedcentral.com/submissions 